\title{
PP28
}

\section{THE VEILING LUMINANCE IN TUNNEL LIGHTING INSTALLATION}

\author{
Giuseppe Rossi et al.
}

DOI 10.25039/x46.2019.PP28

from

CIE x046:2019

Proceedings

of the

29th CIE SESSION

Washington D.C., USA, June 14 - 22, 2019

(DOI 10.25039/x46.2019)

The paper has been presented at the 29th CIE Session, Washington D.C., USA, June 14-22, 2019. It has not been peer-reviewed by CIE.

(C) CIE 2019

All rights reserved. Unless otherwise specified, no part of this publication may be reproduced or utilized in any form or by any means, electronic or mechanical, including photocopying and microfilm, without permission in writing from CIE Central Bureau at the address below. Any mention of organizations or products does not imply endorsement by the CIE.

This paper is made available open access for individual use. However, in all other cases all rights are reserved unless explicit permission is sought from and given by the CIE.

CIE Central Bureau

Babenbergerstrasse 9

A-1010 Vienna

Austria

Tel.: +4317143187

e-mail: ciecb@cie.co.at

www.cie.co.at 


\title{
THE VEILING LUMINANCE IN TUNNEL LIGHTING INSTALLATION
}

\author{
Rossi, G. ${ }^{1}$, lacomussi, P. ${ }^{1}$ \\ 1 INRIM, Torino, ITALY \\ g.rossi@inrim.it
}

DOI 10.25039/x46.2019.PP28

\begin{abstract}
The design of lighting installations in tunnels for motorised traffic requires critical compromises be- tween safety and lighting costs. During daytime the lighting level in the first part of the tunnel shall be high enough to compensate visual adaptation.

The CIE 88 technical report (CIE, 2004), the European technical report CR 14380 (CEN, 2003) and several national standards, like the Italian UNI 11095 (UNI 2019) correlate the lighting level in the threshold zone with the veiling luminance evaluated in the access zone, i.e. in the zone in front of the tunnel portal with a length equal to the stopping distance.

The measurement of the veiling luminance is greatly simplified using Image Luminance Measuring Devices (ILMDs) but its evaluation for the design specification of the lighting installation is a task not adequately described in standards. Some possible approaches are described and compared considering different available data and simplifications.
\end{abstract}

Keywords: Tunnel, lighting

\section{Introduction}

Generally, if the length of tunnel is lower than $1,5 \mathrm{~km}-2 \mathrm{~km}$, the main energy consumption of the lighting installation is concentrate in the first part of the tunnel (the entrance zone). For this reason, its correct design (i.e. the definition of the minimum lighting level required for safety) and real-time control (i.e. the correlation between the actual environmental conditions that influences perception and the actual illumination level) in this zone play a key role in the total cost of the lighting installation and of its management. Therefore the design of lighting installations in tunnels is a critical task because during daytime the lighting level in the first part of the tunnel shall be high enough to compensate the influence the environmental lighting conditions have on the human visual system, i.e. the so called black hole effect and to assure the driver is able to perceive dangerous objects on the road surface in time to stop safely the vehicle.

For this reason, the first part of a tunnel (the entrance zone) is divided into two different zones with different lighting requirements:

- The threshold zone where the obstacle shall be perceived while the driver is outside the tunnel. The driver eyes are adapted at a relatively high luminance level even if the driver is looking at a dark surface inside the tunnel. The glare due the external environmental luminance and the luminance of the atmospheric scattering of light are the main factors that reduce the perceived contrast be- tween object and background. The lighting level shall be high enough to compensate this reduction of the perceived contrast, mathematically modelled using the well-known concept of veiling luminance (Adrian, 1987). The length of the threshold zone is equal to the stopping distance.

- The transition zone where the driver is inside the tunnel, (i.e. he/she is in the threshold zone). There is not influence of glare due to daylight and influence of atmospheric scattering of light can be neglected for the low lighting level if compared to the external level. In this zone thee lighting level should be gradually reduced considering the adaptation time, i.e. the time the visual system requires to adapt himself to the new stimuli conditions. The transition zone stops when the lighting level becomes equal to the internal zone level where 
the driver's eyes are completely adapted to dark conditions and rules similar to these adopted for night-time can be applied.

The stopping distance useful for design, i.e. the minimum distance the driver needs to perceive the presence of the object on the carriage, depends on:

- deterministic parameters like the type of road, its gradient, the friction coefficient tirepavement, the conditions of the road surface (dry or wet), the maximum vehicle velocity permitted on the road;

- statistical parameters like the performance, mass and conditions of the vehicle, the driver reaction time, etc.

For these reasons, the stopping distance is generally defined by national practices or law. In Italy, the law on road constructions (MIT, 2002) defines for evaluating the stopping distance a set of parameters and calculation conditions. The range of values adopted in tunnel lighting (UNI, 2019) goes from $26 \mathrm{~m}$ (urban road with a speed limit of $30 \mathrm{~km} \mathrm{~h}^{-1}$ ) to $358 \mathrm{~m}$ (wet highway with a speed limit of $120 \mathrm{~km} \mathrm{~h}^{-1}$ and a gradient of $-12 \%$ ).

The safety condition implicitly or explicitly standards adopt consist in requiring a road surface average luminance high enough to create a perceived contrast between object and background (the road surface) greater than the threshold value for the given luminance adaptation of the eyes. This requires the definition of four parameters:

- the dimension and shape of the object (target);

- the reflectance of its face the driver views, considered a Lambertian surface;

- a minimum perceived contrast or a safety factor that multiplies the threshold contrast obtained from mathematical models of human vision (KERK, 1987);

- a physical model to evaluate the veiling luminance.

CIE 88 (CIE, 2003) considers as reference object a cube with a $0,2 \mathrm{~m}$ side with a reflectance $\rho$ equal to 0.2 and a minimum contrast equal to $28 \%$. The Italian standard (UNI.2019) considers the same object but with a reflectance $\rho=0.1$ and a minimum contrast equal to $16 \%$. The veiling luminance is obtained considering the luminance of the atmosphere in the $2^{\circ}$ foveal direction of view and the glare from the environment, quantified using the equivalent veiling luminance $L_{\text {seq. }}$. The Italian standard simplifies the original model of CIE 88 considering the windscreen luminance and the glare from the dashboard as an additive value 0.4 times $L_{\text {seq }}$ measured outside the vehicle and assumes the atmospheric transmissivity and the regular transmittance of the windscreen equal to 1 . Considering these conditions the veiling luminance $L_{v}$ is:

$$
L_{v}=L_{\text {seq,e }}+L_{\text {atm }}+L_{\text {ws }}+L_{\text {seq,i }}=1,4 L_{\text {seq,e }}+L_{\text {atm }}
$$

where

$L_{\mathrm{v}} \quad$ is the veiling luminance, in candelas per square metre;

$L_{\text {seq,e }} s$ the equivalent veiling luminance of the environment evaluated without considering the influence of the vehicle, i.e. with driver in the same position but outside the vehicle, in candelas per square metre;

$L_{\text {atm }} \mathrm{s}$ the luminance of the atmosphere in the $2^{\circ}$ cone corresponding to the foveal field of view evaluated without considering the influence of the vehicle (windscreen haze), i.e. with driver in the same position but outside the vehicle, in candelas per square metre;

$L_{w s} \quad$ is the luminance of the windscreen in the $2^{\circ}$ cone corresponding to the foveal field of view of the driver, in candelas per square metre;

$L_{\text {seq,i }}$ is the equivalent veiling luminance due to light inside the vehicle (dashboard, windscreen) evaluated without considering $L_{\text {seq,e, }}$ in candelas per square metre.

While $L_{\text {seq,e }}$ and $L_{\text {atm }}$ changes with climatic conditions, they are deterministic at a given instant and for a given tunnel. $L_{w s}$ and $L_{\text {seq,i }}$ depend on the vehicle types and conditions and their influence can be evaluated using statistical analysis only. With the conventional simplification of the Italian standard, $L_{v}$ becomes formally a characteristic data of the tunnel that can be 
measured or evaluated at every instant. For simplicity when the Italian standard is considered $L$ seq,e is written as $L_{\text {seq. }}$.

With the above conventions, in CIE 88 the safety condition becomes the minimum value the average road surface luminance shall have at the tunnel portal and can be written as:

$$
L_{\text {th }, \mathrm{r}}(x=0)=c\left(q_{\mathrm{c}}\right) L_{\mathrm{v}}\left(x=-d_{\mathrm{s}}\right)
$$

where

$L_{\text {th, } \mathrm{r}} \quad$ is the required minimum average transversal road luminance at the tunnel portal (i.e. at $x=$ $0 \mathrm{~m}$ ), in candelas per square metre;

$c\left(q_{c}\right)$ is a factor that depends on the standard choices about the target and the threshold contrast, in unit 1 ;

$q_{c} \quad$ is the contrast revealing coefficient, i.e. the ratio between the luminance of the road surface and the vertical illuminance $E_{v}$ at the same point, per steradians;

$x \quad$ is the longitudinal coordinate, starting at the tunnel portal and positive in the driving direction, in metres;

$d_{\mathrm{s}} \quad$ is the stopping distance, in metres.

According to the values of $q_{\mathrm{c}}$ the tunnel lighting installations can be classified in symmetric lighting, counter-beam lighting and pro-beam lighting.

There is a formal discrepancy in equation (2). At $x=0 \mathrm{~m}$ the vertical illuminance on the object is due to daylight and not to the artificial light of the lighting installation. In the first meters of the tunnel $(10 \mathrm{~m}-20 \mathrm{~m})$ the daylight contribution on $E_{\mathrm{v}}$ rapidly decrease at negligible level while the artificial light contribution increase. For this reason $q_{\mathrm{c}}$ is evaluated considering only the contribution of the lighting installation in the part of the threshold zone where it becomes stable.

The safety condition of equation (2) can be extended for each section of the threshold zone of the tunnel (i.e. for $0<x \leq d_{s}$ ) as long as the minimum perceived contrast remain constant. This requires the evaluation of $L_{v}$ along the access zone $\left(-d_{\mathrm{s}} \leq x<0\right)$ and the introduction of a new condition to verify the adaptation of the visual system.

When CIE 88 was written, the evaluation of the veiling luminance for all the access zone was considered very complex. Therefore CIE 88 requires the evaluation of $L_{v}\left(x=-d_{s}\right)$ and divides the threshold zone into two parts of equal length. In the first part, the average transversal road luminance shall be equals to $L_{\mathrm{th}, \mathrm{r}}(x=0)$ (equation 2 ). In the second part this value linearly decrease and, at the end of the threshold zone, becomes equal to $L_{\mathrm{th}, \mathrm{r}}\left(x=d_{\mathrm{s}}\right)=0,4 L_{\mathrm{th}, \mathrm{r}}(x=0)$.

The results of measurements on several tunnels $(\mathrm{CIE}, 1984)$ justify this heuristics solution that simplifies the design of tunnel lighting installations but do not optimise the electrical energy consumption. Experiences developed in several countries highlight different approaches able to reduce the installation cost without reducing the traffic safety level (CEN, 2003).

\section{The new Italian standard}

For the threshold zone the new Italian standard (UNI, 2019) suggests the CIE 88 approach for short tunnels, where the stopping distance is greater than the tunnel length or for tunnels with speed limit lower or equal to $50 \mathrm{~km} \mathrm{~h}^{-1}$. For the other tunnels and if the veiling luminance function (see 3 ) is known, the required minimum value of the average transversal road luminance is:

$$
L_{\text {th }, r}(x)=c L_{v}\left(x--d_{s}\right) \text { for } 0 \leq x \leq d_{s}
$$

where

$L_{\text {th, }, r}(x)$ is the required minimum average transversal road luminance at the tunnel section with longitudinal coordinate $x$, in candelas per square metre;

$c$ is a factor that depends on the type of lighting: $c=0,25$ for symmetric lighting, $c=0,23$ for counter-beam lighting and $c=0,32$ for pro-beam lighting, in unit 1 ; 
$x \quad$ is the longitudinal coordinate, starting at the tunnel portal and positive in the driving direction, in metres;

$d_{\mathrm{s}} \quad$ is the stopping distance, in metres.

The above condition is the extension of the safety condition of CIE 88 (equation 2). The second condition:

$$
\frac{L_{\mathrm{th}, \mathrm{r}}\left(x+d_{t}\right)}{L_{\mathrm{th}, \mathrm{r}}(x)} \geq 0,55
$$

where $d_{\mathrm{t}}=0,154 d_{\mathrm{s}}$, requires that the maximum rate of reduction of the luminance in the threshold zone shall be equal or lower than the value defined in CIE 88 for the second part of the threshold zone. This guarantees the same condition of visual adaptation of CIE 88, as required by the Italian law (MIT, 2005), and gives the ratio of the road luminance values between the background viewed at the bottom and at the top of the reference object.

To reduce the electrical energy consumption, the real average transversal road luminance cannot be greater than 1,5 times the minimum required values. To obtain this goal it is necessary to measure in real time the veiling luminance at least at the stopping distance.

\section{The veiling luminance function}

The veiling luminance measured along the access zone is named veiling luminance function $L_{v}(x, t)$. It is a function of the distance from the tunnel portal $x$ and of the time $t$ of evaluation.

When $x$ approaches zero, the veiling luminance becomes very low because the $L_{\text {atm }}$ decreases and $L_{\text {seq }}$ is strongly influenced by the lighting level inside the tunnel. A conventional limit shall be considered to avoid unrealistic values or complex evaluation of $L_{v}$. The Italian standard suggest a limit distance $d_{\text {lim }}$ when the cone with angle of opening of $36^{\circ}$ for the evaluation of $L_{\text {seq }}$ has at least a point in common with the portal hole. For two lines mountain tunnels this correspond at about 3 time the portal height. The last part of the veiling luminance function becomes $L_{v}\left(-d_{\lim } \leq x<0\right)=L_{v}\left(x=d_{\text {lim }}\right)$.

For a given tunnel the veiling luminance function is a stochastic function that depends on the hour of the day, day of the year, and year. It can be defined:

- at a given instant $t$, e.g. if it is measured to control the luminous flux emitted by the luminaries to obtain the right average transversal road luminance values;

- $t$ statistically considering one year period, e.g. to confirm the correct design of the installation;

- as a maximum values to be used for designing the lighting installation and for calculating the maximum installed luminous flux

The last possibility requires some compromises. The veiling luminance can rise at very high values for short periods of time or during peculiar climatic situations. It is not economical to design the lighting installation considering the maximum possible value of the veiling luminance. CIE 88 suggest to use the highest value likely to occur at least 75 daytime hours per years. This solution has the following main objections:

- during $75 \mathrm{~h}$ in a year the lighting conditions in the tunnel could not guarantee adequate safety conditions;

- there is not correlation between the $75 \mathrm{~h}$ and the traffic volume, i.e. the inadequate lighting level could statistically happens when traffic volume conditions create the highest probability of incidents;

- it is difficult to dimostrate that the $75 \mathrm{~h}$ limit is really satisfied by the design choices. What happens if after one year of continuous measurement it is verified that the real limit was 80 $\mathrm{h}$ ?

- as a consequence, during the final testing/commissioning phase there are not standard rules that link measurements with compliance with standard requirements and/or with design expectations. 
The solution generally adopted is to use values given as examples in CIE 88. This solution is adopted also in the Italian standard, if measurement cannot be done. This solution does not resolve the above perplexities, because the proposed values could require correction is some orographic situations, like in mountain tunnels and there is not a clear correlation with the $75 \mathrm{~h}$ requirement.

\section{Measurement of the veiling luminance}

The veiling luminance at a given instant $t$ can be measured using an Image Luminance Measuring Device (ILMD) and the method described in CIE 88 (Adrian diagram) or using the integral equation from with the Adrian diagram was derived (Adrian, 1987). The values and measurement uncertainty of the two methods are comparable but the second approach is more simple if data are elaborated automatically. The important points are the calibration of the ILMD, considering also the angular direction framed by each detector pixels, its dynamic range (linearity between dark and bright zones) and its spectral correction. For continuous measurement the ILMD cannot be positioned in the point the standard requires. This problem can be solved considering a set measurement as described in EN 13201-4 (CEN, 2015) for road lighting system. During the set measurement two ILMDs are used. The first one is a reference system aligned in the correct position, the second one is the measured system installed in a fixed position, e.g. in a column at the kerbside. Comparing the two measures at the same instant a sort of on-site calibration is carried out. The correction is not constant with time, but in tunnel with straight access road discrepancies are acceptable and comparable with the measurement uncertainty of these instruments $(5 \%-8 \%)$. A more accurate correction can be done using photogrammetry methodologies considering that during the set measurement, the tunnel portal is framed from two different points of view. The correction considers the framed surfaces as lambertian diffusers and modifies the weighting each pixel have with directions. This methodology requires a modification of the firmware of the ILMD but can drastically reduce the influence of the incorrect position of the instrument.

The measurement of the veiling luminance function can be done with a mobile laboratory as a sequence of $L_{v}$ measurements approaching the tunnel portal. The Italian standard requires at least 3 measures and a linear approximation between points, but a step of $5 \mathrm{~m}$ is commonly used, except when manual system are adopted. This limit is a compromise considering also the situation when the design of the lighting system shall be done while the tunnel is under construction and measurements are difficult.

A possible alternative technique uses the frame acquired at the stopping distance and changes the angular value of each pixel to simulate the different distances from the tunnel portal. In tunnel with straight access road, generally this technique introduces differences respect to the correct measured values lower than $5 \%$, but this figure greatly changes from tunnel to tunnel. The variation of the atmospheric luminance with distance cannot be measured with this technique, and a linear decrement is usually considered.

The measurement of $L_{v}$ at a given instant $t$ is useful for controlling the lighting installation or for verifying its correct operation but does not give information of the effective performance of the installation without other data.

The solution is to measure also some other environmental parameters able to give a correlation between the measured veiling luminance and the valued that shall be used for design. The following procedure is under testing for some Italian tunnels that have installed permanently an ILMD for controlling the lighting installation. The results will be available after one year of testing.

During the measurement of the veiling luminance carried out with a mobile laboratory the horizontal illuminance is measured and the sky condition are recorded considering the CIE classification (CIE, 2014). The measurement of the horizontal illuminance can be done with a illuminance meter on the top of the vehicle and the sky condition can be recorded using a second ILMD with a wide-angle lens. With this data it is possible to evaluate the vertical illuminance on the tunnel portal and the veiling luminance with different illumination levels and sky conditions. Inter-reflection between lighted surfaces could be important but, as a first approximation, their contribution can be considered proportional to horizontal luminance, and 
therefore considered in the measurement. A Montecarlo statistical analysis adapting the tolerance methodology described in (CEN, 2015) to this situation can produce a probability density function of the veiling luminance values. The design value can be selected considering the corresponding distribution function, for example the veiling luminance that gives a coverage probability of less than $95 \%$.

To improve the evaluation accuracy, measurements can be carried out in different seasons if the orography behind the portal changes (e.g. snow during the winter and green grasses during summer). The presence of sun in the visual field used for veiling luminance calculation (a cone with an angle of opening of $56,8^{\circ}$ ) can be verified using free tools available on-line for evaluating the daily and hourly position of the sun. The presence of the sun increases the veiling luminance at a level that suggest the use of additional shading settings such as grille entrances.

A completely different approach starting from the measurement of the veiling luminance and horizontal illuminance is to correlate these data with table used for evaluation of energy need for buildings heating and cooling. For example the Italian standard UNI TR 10349 (UNI, 2008) gives formula and methods for splitting global solar irradiance into the direct and diffuse parts and for calculate the solar irradiance on tilted and vertical planes. The standard gives tables of solar irradiance data for 102 Italian localities, considering the average value for each months of the year. From these values it is possible to calculate the monthly average value of illuminance using the solar luminous efficacy value of $94,2 \mathrm{Im} \mathrm{W} \mathrm{m}^{-1}$. Using these data and the measurement results, the greatest value of the average monthly veiling luminance can be obtained. Adding a safety factor conventionally introduced by standard, the value of the veiling luminance to be accepted in design can be defined.

This approach is interesting because with a single measurement and the use of standard data, without the tedious calculation of the alternative procedure proposed, gives a result without any other verification like the actual method of the $75 \mathrm{~h}$ formally requires.

\section{Conclusion}

Two different methods that can be adopted in design optimised tunnel lighting installation are proposed. The possible energy saving justify the greater effort to obtain more detailed data and information before defining the characteristic of a tunnel lighting installation. The methods will be compared using data acquired during summer in several Italian tunnel equipped with ILMD for the veiling luminance real time measurement.

\section{References}

ADRIAN, W. 1987. Adaptation luminance when approaching a tunnel in daytime. Lighting Res. Technol., 19, 73-79.

CEN 2003. CEN CR 14389:2003. Lighting applications - Tunnel lighting. Brussels: CEN.

CEN 2015. CEN EN 13201-4:2015. Road lighting - Part 4: Methods of measuring lighting performance. Brussels: CEN.

CIE 1984. CIE 61:1984. Tunnel entrance lighting: a survey of fundamentals for determining the luminance in the threshold zone. Vienna: CIE.

CIE 2004. CIE 88:2004. Guide for the Lighting of Road Tunnels and Underpasses. Vienna: CIE.

CIE 2014. CIE 215:2014. CIE Standard General Sky Guide. Vienna: CIE.

KERK, M. 1987. Effect of luminaire arrangement on object visibility. TRANSPORTATION RESEARCH RECORD, 1247, 17-22.

MIT 2005. MIT 4 gennaio 2002. Norme funzionali e geo- metriche per la costruzione delle strade. Roma: MINISTERO DEI TRASPORTI E DELLE INFRASTRUTTURE.

MIT 2005. MIT 20 dicembre 2005. Norme di illuminazione delle gallerie stradali. Roma: MINISTERO DEI TRASPORTI E DELLE INFRASTRUTTURE. 
UNI 2016. UNI 10349-1:2016. Riscaldamento e raffrescamento degli edifici- Dati climatici -Parte 1: Medie mensili per la valutazione della prestazione termo-energetica dell'edificio e metodi per ripartire l'irradianza solare nella frazione diretta e diffusa e per calcolare l'irradianza solare su di una superficie inclinata. Milano: UNI.

UNI 2019. UNI 11095:2019. Luce e illuminazione - Illuminazione delle gallerie stradali. Milano: UNI. 